Communications in Physics, Vol.26, No. 1 (2016), pp. 83-92

DOI:10.15625/0868-3166/26/1/8287

\title{
ANALYTICAL POSSIBILITIES OF RUTHERFORD BACKSCATTERING SPECTROMETRY AND ELASTIC RECOIL DETECTION ANALYSIS METHODS
}

\author{
VU DUC PHU AND LE HONG KHIEM ${ }^{\dagger}$ \\ Institute of Physics, Vietnam Academy of Science and Technology, \\ 10 Dao Tan, Ba Dinh, Hanoi, Vietnam
}

\author{
A. P. KOBZEV AND M. KULIK ${ }^{\star}$ \\ Frank Laboratory of Neutron Physics, Joint Institute for Nuclear Research, \\ 141980 Dubna, Russia \\ ${ }^{\star}$ Institute of Physics, Maria Curie-Skłodowska University, \\ pl. M. Curie-Sktodowskiej 1, 20-031 Lublin, Poland \\ ${ }^{\dagger}$ E-mail:1hkhiem@iop.vast.vn
}

Received 29 April 2016

Accepted for publication 21 June 2016

\begin{abstract}
This paper presents the results of an experimental study of three samples containing various elements in the near-surface layers. The depth profiles of all the elements of different atomic masses from hydrogen to silver were investigated by Rutherford Backscattering Spectrometry (RBS) and Elastic Recoil Detection Analysis (ERDA). The experiments were performed by using the low-energy (about $2 \mathrm{MeV}$ ) ${ }^{4} \mathrm{He}^{+}$ion beams. The obtained results demonstrate the possibility of the RBS and ERDA methods in the investigation of depth profiles of any mass element with an atomic concentration of about 0.01 at.\% and a depth resolution around $10 \mathrm{~nm}$.
\end{abstract}

Keywords: Rutherford backscattering spectrometry; elastic recoil detection analysis; depth profile; ion beam analysis.

Classification numbers: 01.30.Rr; 07.77.Ka; 82.80.Yc.

\section{INTRODUTION}

The RBS and ERDA methods are widely used for solving various scientific and applied problems [1-4]. The basic physical principles underlying the RBS and ERDA methods will be presented in the next parts of this paper. The implementation of these methods requires a monochromatic beam of low-energy charged particles. A beam of helium ions generated by an electrostatic generator is best suited for this purpose. In the case of RBS, the ions are observed (C)2016 Vietnam Academy of Science and Technology 
which are ejected from the target by the projectile while in the case of ERDA the object of detection are the projectiles backscattered from the target ions. A highly monochromatic beam is required for the precise identification of the mass of atoms in the samples under study. It is also necessary to record the energy of scattered ions and recoil nuclei with high accuracy. In this study, the EG-5 accelerator that produces a $2 \mathrm{MeV}$ helium ion beam with an energy spread of less than $0.5 \mathrm{keV}$ has been used. The surface-barrier detectors with an energy resolution of $12 \mathrm{keV}$ used in our experiments provide the required accuracy of the measurement of particle energy.

Both RBS and ERDA methods are non-destructive because the ion beam intensity, which is required to achieve the desired statistical accuracy in the spectrum of scattered ions, is normally less than $1 \mu \mathrm{A}$. At the same time, the sufficient intensity of the scattered beam is achieved through a large scattering cross section of helium ions from all elements for the energy near the maximum braking power (Bragg peak). A relatively low energy of a helium ion beam also ensures a high resolution (about $10 \mathrm{~nm}$ ) in studying depth profiles of elements due to high energy losses of helium ions.

\section{RBS METHOD}

The RBS method is the most commonly used and known technique in the field of Ion Beam Analysis for material characterization. The quantitative determination of the composition of a material and depth profiling of individual elements can be obtained by this method without the need for reference samples. The method has a very good sensitivity for heavy elements. The drawback of RBS is the low sensitivity for light elements. Therefore the combination of other ion beam analysis methods is often required and the ERDA method is one of them. A detailed description of the RBS method can be found in many text books and papers, for example in Refs. [1,2].

The basis of this technique is the classical theory of interaction of two charged particles. The interaction between an accelerated ion and a nucleus of the atom that the studied sample consists of can be regarded as the Coulomb force arising in the elastic collision of two charged particles. The scheme of this process is presented in Fig. 1. The incident charged particle with the mass $M_{1}$ and the velocity $V_{0}$ (kinetic energy $E_{0}$ ) interacts with an atomic nucleus with the mass $M_{2}$, which is at rest before the interaction. As a result of this interaction, the incident particle is scattered at an angle $\theta$, and its velocity decreases to the value $V_{1}$ (kinetic energy is $E_{1}$ ). The atomic nucleus also acquires the velocity $V_{2}$ (kinematic energy $E_{2}$ ) and moves at an angle $\phi$ with respect to the initial trajectory of the bombarding particles.

Using the laws of conservation of energy and momentum, we can obtain an expression for the kinematic factor $K$, which allows us to calculate the energy of the scattered particle by the following formula [1]:

$$
K \equiv \frac{E_{1}}{E_{0}}=\left[\frac{M_{1} \cos \theta+\sqrt{M_{2}^{2}-M_{1}^{2} \sin ^{2} \theta}}{M_{1}+M_{2}}\right]^{2}
$$

It can be seen from the formula (1) that only particles with a mass less than the mass of the scattering atom can be backscattered. Therefore, it is impossible to investigate depth profiles 
of hydrogen isotopes in the near-surface layers of samples using the RBS method. The ERDA technique is usually used to study depth profiles of hydrogen isotopes.

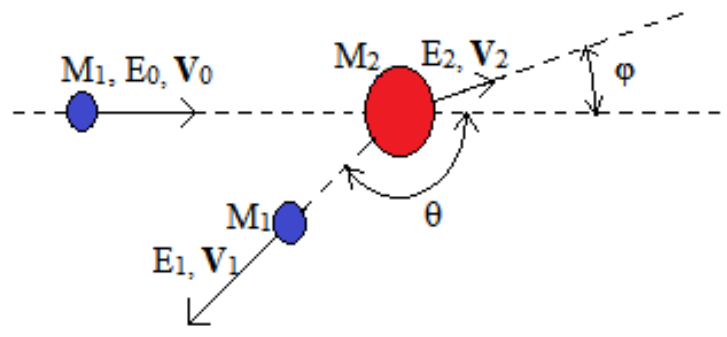

Fig. 1. Diagram of interaction of a moving ion with an atomic nucleus.

It should also be noted that the kinematic factor increases with respect to increase of the mass of scattering atom, but it reaches constant values for very heavy elements. This makes the identification of the heavy elements more difficult. The RBS technique also allows one to determine the concentration of elements in the near-surface layers of the sample under investigation. To perform the calculations, it is necessary to know the scattering cross section of the bombarding particles from the atoms of the sample. In most cases the Rutherford formula is used for the calculations, that is why this analytical technique got the name "Rutherford back scattering method". In the laboratory frame the Rutherford formula is given by the following expression [1]:

$$
\frac{d \sigma}{d \Omega}=\left(\frac{Z_{1} Z_{2} e^{2}}{4 E}\right)^{2} \frac{4}{\sin ^{4} \theta} \frac{\left\{\cos \theta+\left[1-\left(M_{1} / M_{2} \sin \theta\right)^{2}\right]^{1 / 2}\right\}^{2}}{\left[1-\left(M_{1} / M_{2} \sin \theta\right)^{2}\right]^{1 / 2}}
$$

where $Z_{1}$ is the atomic number of the accelerated particle, and $Z_{2}$ is the atomic number of the element in the target.

It is clear from equation (2) that the scattering cross section grows as $\mathrm{Z}^{2}$, which increases the sensitivity of the method to heavier elements. In addition, the scattering cross section decreases with increasing energy of the incident particle as $E^{-2}$. This is the reason for using a particle beam of low energy for the RBS method.

The recorded RBS spectra can be transformed to the compositional depth profile of elements contained by the sample using the computer program SIMNRA [5]. It is noted that the determination of energy loss by scattered particles is an important factor in calculating the element depth profiles. Typically, the energy of a charged particle at a depth $x$ in solids is calculated as follows [1]:

$$
E=E_{0}-\int_{0}^{x}(d E / d x) d x
$$

The value $(d E / d x)$ is the energy losses of charged particles per unit path length in the medium. As is shown in Fig. 2, the incident particle experiences a loss of energy both before the 
scattering from the atom located at a depth $x$ and after the scattering. The energy of the scattered particles entering the detector is calculated using the energy loss factor $[S][1]$ :

$$
[S] \equiv\left[\left.\frac{K}{\cos \theta_{1}} \frac{d E}{d x}\right|_{\text {in }}+\left.\frac{1}{\cos \theta_{2}} \frac{d E}{d x}\right|_{\text {out }}\right]
$$

The energy of the particle recorded by the detector after scattering at a depth $x$, is equal to:

$$
E_{1}=E_{0}-[S] x
$$

The RBS method for calculating the energy losses in the substance layer uses the following formula for the stopping cross section factor:

$$
[\varepsilon]=[S] / N
$$

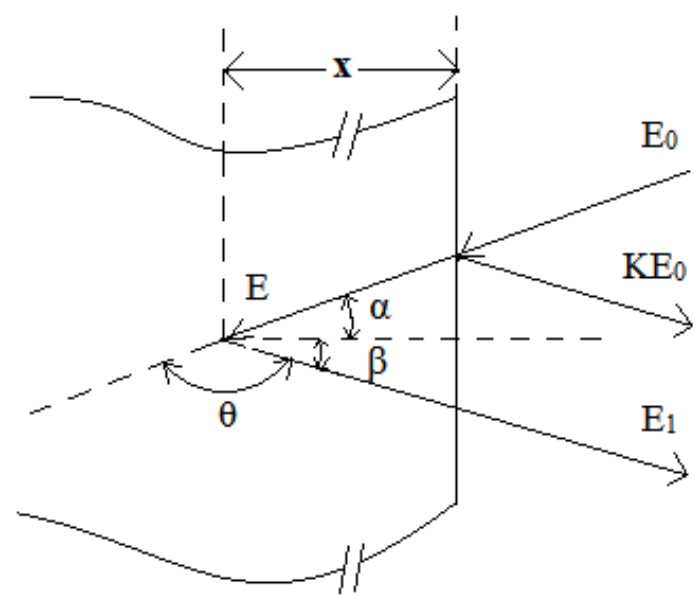

Fig. 2. Diagram of energy losses for the particle scattered at a depth $x$.

If the studied sample has a complex chemical composition then the stopping cross section factor can be calculated using the Bragg's rule. For a molecular substance consisting of two elements with atomic numbers $\mathrm{A}$ and $\mathrm{B}$, and the concentrations of these atoms are $m$ and $n$, respectively, the Bragg's rule gives the following value of the stopping cross section factor [1]:

$$
\varepsilon^{A_{m} B_{n}}=m \varepsilon^{A}+n \varepsilon^{B},
$$

where $\varepsilon^{A}$ and $\varepsilon^{B}$ are the stopping cross section factors for homogeneous materials $A$ and $B$, respectively.

The height of a single channel of the RBS energy spectrum can be calculated as follows [1]:

$$
N=\sigma\left(E_{0}\right) Q \Omega \Delta /[S] \cos \beta,
$$

where $\sigma\left(E_{0}\right)$ is the differential scattering cross section for energy $E_{0} ; Q$ is the number of charged particles incident on the surface of the analyzed sample during the exposure; $\Omega$ is the solid angle of the scattered particle detector; $\beta$ is the angle between the beam direction and the normal to the sample surface; $\Delta$ is the energy width of a channel of the spectrometer. 
Thus, by measuring the spectrum of scattered helium ions, we examine the depth profiles of all elements, starting from the lightest (carbon) to the heaviest elements that are found in the structures having a scientific or technical application. More accurate results are obtained when heavy elements are in the composition of a thin surface layer on a solid substrate of lighter elements. In the opposite case, helium ions scattered by the atoms of light elements are superimposed on the spectrum obtained from a heavy substrate, and this somewhat reduces the accuracy of determination of concentration of light elements. For the determination of the element content in the surface layer it is necessary to carry out the energy calibration of the spectrometer. For the energy calibration, two known elements contained in the surface layer of the sample can be used to calculate the channel width and zero displacement. The energy of the detected particle is linearly proportional to the channel number of the multichannel amplitude analyzer. In the RBS technique, a standard sample containing at least two elements on its surface can be used for the energy calibration. The accurate calibration makes it possible to determine the content of different elements from carbon to niobium. But the depth profiles of hydrogen isotopes can be measured only using a special ERDA technique.

\section{ERDA METHOD}

The ERDA is an Ion Beam Analysis technique used for material characterization for obtaining elemental concentration depth profiles in material samples. The method is very similar to RBS, but instead of detecting the projectile at the back angle, the recoils are detected in the forward direction. In the ERDA method, an energetic ion beam from an accelerator is used for irradiation of a sample to be examined. The ions of beam will interact with the atoms of the sample and such interactions are commonly of Coulomb nature. The method is often used for the elemental analysis and information about the depth profile of the sample can be obtained by the ERDA method. The advantage of the ERDA in comparison with RBS is that the ERDA has enabled depth profiling of elements from lightest elements like hydrogen up to heavy elements with high resolution in the light mass region.

The scheme of the process of production of recoil nuclei as a result of an elastic collision of two charged particles is presented in Fig. 3. The recoil nuclei move at different angles $\phi<90^{\circ}$. In ERDA experiments we usually use a ${ }^{4} \mathrm{He}^{+}$ion beam with an energy of $2297 \mathrm{keV}$ [6, 7]. A silicon surface-barrier detector is placed at an angle of $30^{\circ}$ with respect to the direction of incident particles. The incident angle is usually $75^{\circ}$ in all ERDA experiments. Normally, the sample under study comprises both light and heavy elements, and helium ions scattered by heavy elements at an angle $\phi$ create a high background for an ERDA detector. A thin aluminum foil is placed in front of the detector to stop scattered helium ions. The thickness of the foil is chosen in such a way that it should be sufficient to absorb scattered He ions, but must ensure passing of recoil $\mathrm{H}$ and $\mathrm{D}$ ions with minimal energy losses. The energy calibration of an ERDA spectrometer can be performed easily, if there are two hydrogen isotopes in the surface layer of the sample under study. If there is only one hydrogen isotope in the surface layer, it is necessary to obtain the second spectrum at energy of $2035 \mathrm{keV}$. To investigate the depth profiles of all elements in the surface layers for different samples, one can use both RBS and ERDA methods simultaneously. In our experiment the second silicon surface-barrier detector was placed at $\theta=120^{\circ}$. The combined use of the RBS and ERDA methods improves the accuracy of the measurement of element depth profiles 
for multi-element samples. The geometrical arrangement is chosen to ensure the sensitivity of the measurement to be the highest possible.

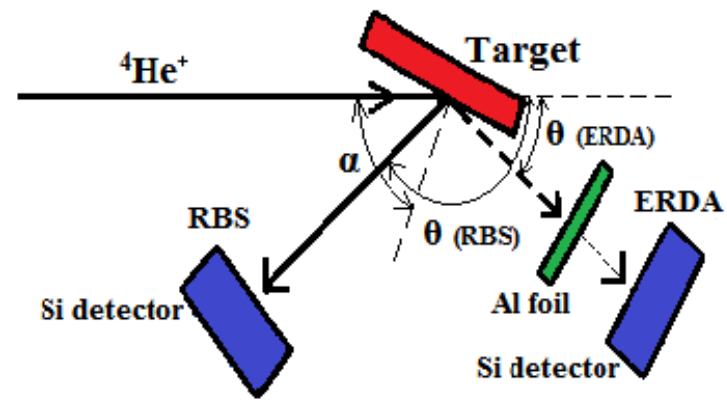

Fig. 3. Layout of the experiment for the combined use of the RBS and ERDA methods.

\section{EXPERIMENTAL RESULTS}

Three Si samples with various elements in the near-surface layers were used to demonstrate the possibilities of the RBS and EDRA methods. Si samples are of importance in microelectronics. These samples consist of a Si substrate and a thin layer with other elements. The first sample was used to demonstrate the possibilities of the RBS method. An RBS spectrum was obtained under the following experimental conditions: $E=2046 \mathrm{keV}$, $\theta=170^{\circ}, \alpha=30^{\circ}$. Fig. 4 presents a spectrum of scattered helium ions which was obtained for sample o․ Fig. 4 also shows a simulated spectrum calculated using the SIMNRA software. The number of particles incident on the surface of the sample during the exposure time is taken to be the one that provides the good

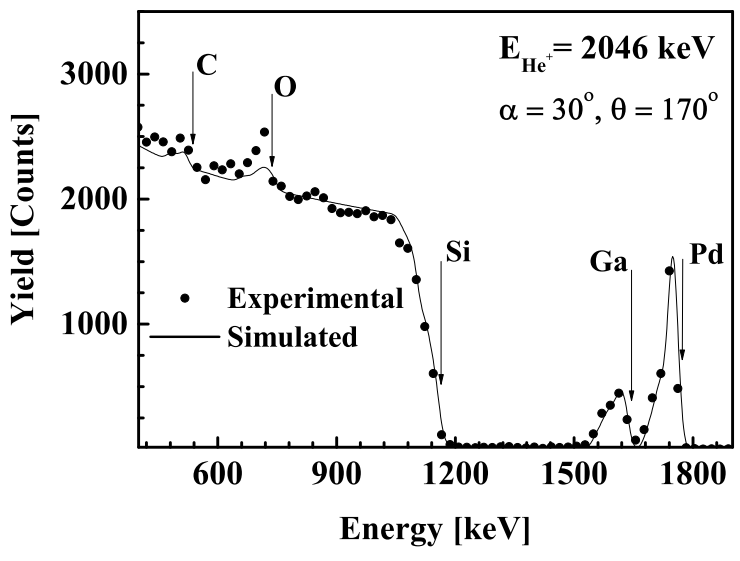

Fig. 4. Experimental and simulated RBS spectra for sample אo 1. agreement between the simulated and experimental spectra in the region where the scattering from a silicon substrate is observed. As may be seen from Fig. 4, no admixtures are present in the silicon substrate, but there are various elements $(\mathrm{C}, \mathrm{O}, \mathrm{Ga}, \mathrm{Pd})$ in the near-surface layer.

The depth profiles of all elements in sample 1 are presented in Table 1. The surface layer of the silicon sample about $200 \mathrm{~nm}$ thick comprises two light elements (C, O) and two heavy elements $(\mathrm{Ga}, \mathrm{Pd})$. The concentration of oxygen and carbon changes with depth from 10 at.\% to 30 at. $\%$ and from 20 at. $\%$ to 60 at.\%, respectively. The concentration of gallium decreases with depth from 5.5 at.\% to 1.8 at.\% and the concentration of palladium has a maximum of 13 at.\% at a depth of about $40 \mathrm{~nm}$ and decreases down to 1.3 at.\% towards the bottom of the layer. The 
above-listed concentrations show a good sensitivity of the RBS method to various elements at a depth resolution of about $10 \mathrm{~nm}$.

Table 1. Depth profiles of all elements in sample אol.

\begin{tabular}{|c|c|c|c|c|c|c|c|}
\hline \multirow{2}{*}{ Layer number } & \multicolumn{3}{|c|}{ Thickness } & \multicolumn{5}{|c|}{ Concentration of each element (in unit \%) } \\
\cline { 2 - 8 } & $\mathbf{1 0}^{15}$ atoms/cm & $\mathbf{n m}$ & $\mathbf{C}$ & $\mathbf{O}$ & $\mathbf{S i}$ & $\mathbf{G a}$ & Pd \\
\hline $\mathbf{1}$ & 130 & 20.3 & 56.0 & 16.5 & 20.0 & 5.5 & 2.0 \\
\hline $\mathbf{2}$ & 150 & 25.2 & 22.0 & 24.5 & 35.0 & 5.5 & 13.0 \\
\hline $\mathbf{3}$ & 150 & 38.3 & 20.0 & 28.8 & 45.0 & 3.7 & 2.5 \\
\hline $\mathbf{4}$ & 250 & 37.1 & 22.0 & 21.9 & 50.0 & 3.3 & 2.8 \\
\hline $\mathbf{5}$ & 250 & 45.9 & & 11.9 & 85.0 & 1.8 & 1.3 \\
\hline $\mathbf{6}$ & 200 & 40.0 & & 10.0 & 90.0 & & \\
\hline $\mathbf{7}$ & 8000 & & & & 100.0 & & \\
\hline
\end{tabular}

Sample $\_2$ was investigated using simultaneously two analytical RBS and ERDA techniques. The experimental conditions for the ERDA method are the same as usual and the experimental conditions for RBS are shown in Fig. 5. This figure also shows an RBS spectrum measured for sample $\_2$. The surface layer of sample $\_2$ comprises both light $(\mathrm{H}, \mathrm{C}, \mathrm{O})$ and heavy elements $(\mathrm{Ni}, \mathrm{Ag})$. Using the RBS method, the depth distributions of nickel and silver were measured with high accuracy.

The atomic concentration of nickel and silver changes from 10 at. $\%$ to 28 at. $\%$ and from 1.0 at.\% to 1.7 at.\%, respectively in the layer with a thickness of about $47 \mathrm{~nm}$. The change of the atomic concentration of silicon

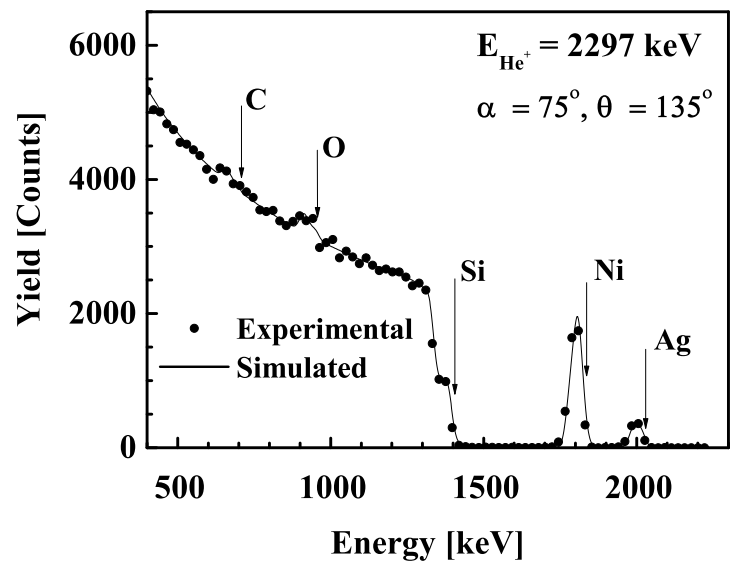

Fig. 5. Experimental and simulated RBS spectra for sample ֵo2. within $4.0-44.0$ at.\% in the layer $257 \mathrm{~nm}$ thick was measured with high accuracy as well. However, the accuracy of the measurement of concentrations of carbon and oxygen was not so high for the same reasons as in the case of sample 1 (overlapping of the spectrum of helium ions scattered by these elements and the spectrum of particles scattered by a silicon substrate and small scattering cross sections for carbon and oxygen). The depth profiles of all elements in sample 2 are presented in Table 2. 
Table 2. Depth profiles of all elements in sample 츤2.

\begin{tabular}{|c|c|c|c|c|c|c|c|c|}
\hline \multirow{2}{*}{ Layer } & \multicolumn{2}{|l|}{ Thick } & \multicolumn{6}{|c|}{ Concentration of each element (in unit at.\%) } \\
\hline & $10^{15}$ atoms $/ \mathbf{c m}^{2}$ & $\mathbf{n m}$ & $\mathbf{H}$ & $\mathbf{C}$ & $\mathbf{O}$ & Si & $\mathbf{N i}$ & Ag \\
\hline 1 & 50 & 6.1 & 25.0 & 21.0 & 21.0 & 4.0 & 28.0 & 1.0 \\
\hline 2 & 50 & 6.6 & 15.0 & 22.0 & 22.0 & 12.0 & 28.0 & 1.0 \\
\hline 3 & 100 & 14.2 & 13.0 & 10.8 & 10.5 & 44.0 & 20.0 & 1.7 \\
\hline 4 & 100 & 20.0 & 15.0 & 21.8 & 20.0 & 32.0 & 10.0 & 1.2 \\
\hline 5 & 250 & 45.0 & 22.0 & 30.0 & 25.0 & 23.0 & & \\
\hline 6 & 350 & 71.0 & 15.0 & & & 85.0 & & \\
\hline 7 & 600 & 94.0 & 1.0 & & & 99.0 & & \\
\hline 8 & $5000+$ & & & & & 100.0 & & \\
\hline
\end{tabular}

The depth profile of hydrogen atoms was measured with high accuracy using the ERDA technique. The experimental layout is shown in Fig. 3. The spectrum of recoil protons is given in Fig. 6. The change of the concentration of hydrogen in the layer within 1.0 - 25.0 at.\% was measured rather accurately. The final calculated curves of the RBS and ERDA spectra were obtained by varying the concentration of all elements in each layer of the sample model.

The possibility of studying depth profiles of two hydrogen isotopes ( $\mathrm{H}$ and $\mathrm{D}$ ) was demonstrated in the experiment with sample 3, in which ERDA and RBS spectra

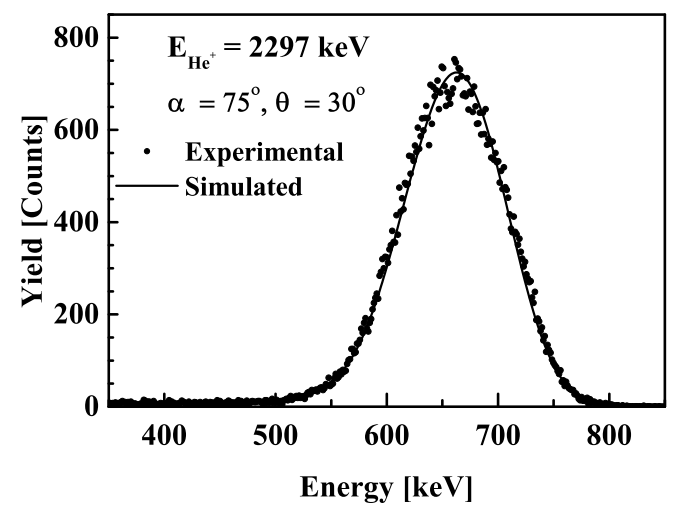

Fig. 6. Experimental and simulated ERDA spectra for sample §o2. were collected simultaneously. In the RBS spectrum (Fig. 7) several heavy elements (Ti, Fe, Mo, Te) whose concentration varied in the range from 0.2 at.\% to 5.0 at.\% were found in the surface layer approximately $110 \mathrm{~nm}$ thick. The basic structure of this layer consists of light elements $(\mathrm{H}, \mathrm{D}, \mathrm{C}, \mathrm{O})$. There are no silicon atoms in this layer, which is confirmed by a shift of the silicon kinematic border in the RBS spectrum.

Fig. 8 presents an ERDA spectrum measured for sample 3. There are two peaks corresponding to recoil nuclei of hydrogen and deuterium. In this spectrum the kinematic borders of both hydrogen isotopes are indicated by arrows. The concentration of deuterium in the layer about $220 \mathrm{~nm}$ thick proved to be rather high $30-50$ at.\%. 


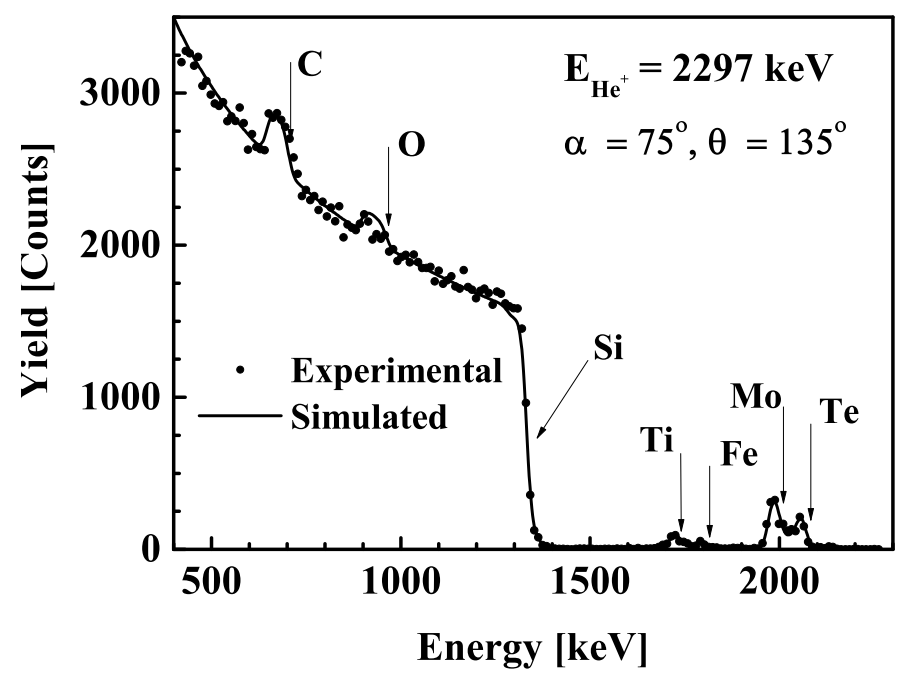

Fig. 7. Experimental and simulated RBS spectra for sample ㄲo 3 .

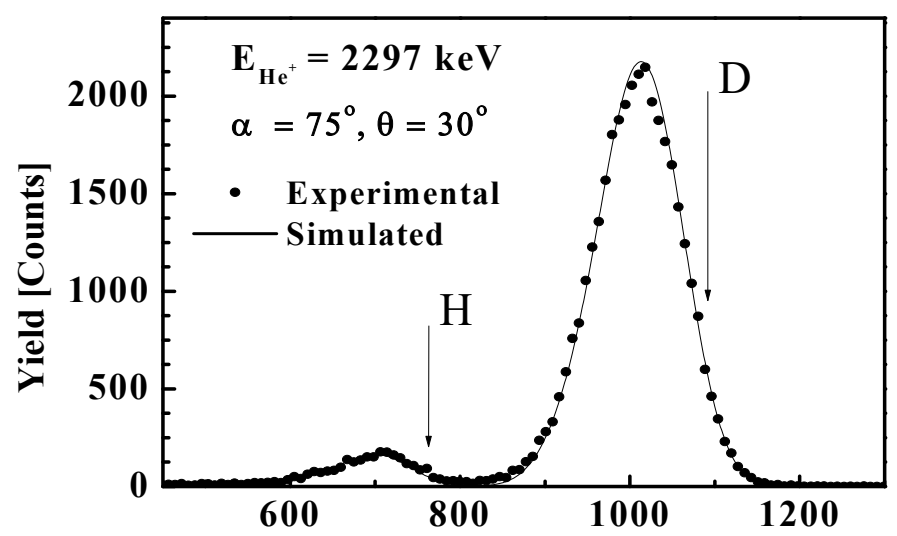

Fig. 8. Experimental and simulated ERDA spectra for sample אํㅡ.

For this thickness of the layer the spectrum of recoil deuterons does not yet overlap the spectrum of recoil protons. This result suggests that it is possible to study the depth profiles of these isotopes in the near-surface layers with a thickness of up to $400 \mathrm{~nm}$. Full information about the depth distributions of all elements in sample $₫ o 3$ is presented in Table 3.

To demonstrate the analytical capabilities of the RBS and ERDA techniques we used the special selected samples with layers of a complex elemental composition deposited on silicon substrates. The above-mentioned techniques may be used only for the elemental analysis of nearsurface layers with a thickness of less than $1 \mu \mathrm{m}$. It is apparent that the characteristics of the 
Table 3. Depth concentrations of elements in sample šo3.

\begin{tabular}{|c|c|c|c|c|c|c|c|c|c|c|c|}
\hline \multirow{2}{*}{ Layer } & \multicolumn{3}{|c|}{ Thick } & \multicolumn{7}{|c|}{ Concentration of each element (in unit \%) } \\
\cline { 2 - 14 } & $\begin{array}{c}\mathbf{1 0}^{15} \\
\text { atoms/cm }\end{array}$ & $\mathbf{n m}$ & $\mathbf{H}$ & $\mathbf{D}$ & $\mathbf{C}$ & $\mathbf{O}$ & $\mathbf{S i}$ & $\mathbf{T i}$ & $\mathbf{F e}$ & $\mathbf{M o}$ & $\mathbf{T e}$ \\
\hline $\mathbf{1}$ & 50 & 7.9 & 30.0 & 31.0 & 26.0 & 5.0 & & 5.0 & 0.8 & 1.5 & 0.7 \\
\hline $\mathbf{2}$ & 50 & 8.1 & 25.0 & 32.0 & 26.4 & 12.0 & & 1.8 & 0.5 & 1.6 & 0.7 \\
\hline $\mathbf{3}$ & 150 & 22.4 & 3.0 & 40.0 & 41.6 & 11.0 & & 1.1 & 0.5 & 2.1 & 0.7 \\
\hline $\mathbf{4}$ & 500 & 71.1 & 2.0 & 42.0 & 43.4 & 12.0 & & 0.2 & 0.2 & & 0.2 \\
\hline $\mathbf{5}$ & 350 & 51.6 & 2.0 & 50.0 & 36.0 & 11.0 & 1.0 & & & & \\
\hline $\mathbf{6}$ & 350 & 71.6 & 2.0 & 20.0 & & & 78.0 & & & & \\
\hline $\mathbf{7}$ & $5000+$ & & & & & & 100.0 & & & & \\
\hline
\end{tabular}

analytical methods presented in this paper significantly deteriorate for samples prepared on substrates comprising elements heavier than silicon. Silicon structures are now widely used in various fields of engineering, which make the RBS and ERDA techniques rather popular.

\section{CONCLUSIONS}

In the present paper we described several typical examples, in which the RBS and EDRA methods were used to study the near-surface layers of solids. The spectra were obtained by using helium ion beams with an energy of about $2-3 \mathrm{MeV}$ from an electrostatic accelerator. The combined use of two methods allows one to investigate the depth distributions of all elements in the surface layer of samples. The accuracy of the determination of the atomic concentration for comparatively light elements is $1-2$ at.\% and achieves 0.01 at.\% for comparatively heavy elements. The depth resolution for the combined use of these two methods is about $10 \mathrm{~nm}$. The maximum depth of the analysis is less than $1 \mu \mathrm{m}$ when a helium ion beam is used in the specified energy range.

\section{REFERENCES}

[1] W.-K. Chu, J. W. Mayer, and M.-A. Nicolet, Backscattering Spectroscopy, Academic Press, New York, 1978.

[2] A. Kobzev, E. Kravtcov, L. Romashev, A. Semerikov, and V. Ustinov, Vacuum 63 (4) (2001) 501-505.

[3] E. Rauhala, N. Barradas, S. Fazinic, M. Mayer, E. Szilágyi, and M. Thompson, Nuclear Instruments and Methods in Physics Research Section B: Beam Interactions with Materials and Atoms 244 (2) (2006) 436-456.

[4] D. Machajdı, A. P. Kobzev, K. Fröhlich, et al., Vacuum 70 (2) (2003) 313-317.

[5] M. Mayer, Simnra User's Guide, http:rzg.mpg.de/ mam.

[6] L. Hrubčin, J. Huran, R. Šandrik, A. Kobzev, and D. Shirokov, Nuclear Instruments and Methods in Physics Research Section B: Beam Interactions with Materials and Atoms 85 (1) (1994) 60-62.

[7] A. Kobzev, J. Huran, D. Maczka, and M. Turek, Vacuum 83 (2009) S124-S126. 